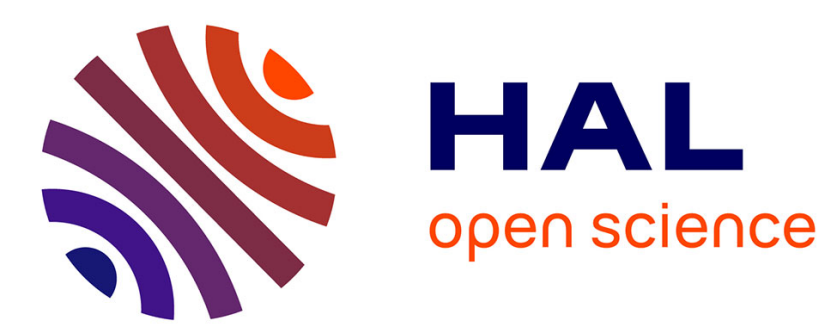

\title{
The theory of elastic and viscoelastic micropolar liquids
}

\author{
V. A. Yeremeyev, L. M. Zubov
}

\section{To cite this version:}

V. A. Yeremeyev, L. M. Zubov. The theory of elastic and viscoelastic micropolar liquids. Journal of Applied Mathematics and Mechanics, 1999, 63 (5), pp.755-767. hal-00835631

\section{HAL Id: hal-00835631 https://hal.science/hal-00835631}

Submitted on 19 Jun 2013

HAL is a multi-disciplinary open access archive for the deposit and dissemination of scientific research documents, whether they are published or not. The documents may come from teaching and research institutions in France or abroad, or from public or private research centers.
L'archive ouverte pluridisciplinaire HAL, est destinée au dépôt et à la diffusion de documents scientifiques de niveau recherche, publiés ou non, émanant des établissements d'enseignement et de recherche français ou étrangers, des laboratoires publics ou privés. 


\title{
THE THEORY OF ELASTIC AND VISCOELASTIC MICROPOLAR LIQUIDS
}

\author{
V. A. YEREMEYEV and L. M. ZUBOV \\ Rostov-on-Don (e-mail: eremeyev@math.rsu.ru, zubov@math.rsu.ru)
}

\begin{abstract}
A model of a liquid medium with couple stresses, which generalizes the theory of a viscous micropolar liquid to the same degree with which the constitutive relations of a simple viscoelastic liquid generalize the equations of a state of a Ncwtonian liquid, is proposed. The similarity and distinction between the model of an elastic micropolar liquid and the model of a medium equipped with a director field and used to describe nematic liquid crystals is discussed. Problems of the equilibrium of a micropolar liquid, including the problem with a free surface are considered. The equilibrium conditions of the phases of an elastic micropolar liquid are derived by a variational method. Problems of the flow of a viscoelastic liquid are derived by a variational method. Problems of the flow of a viscoelastic liquid in a circular tube and between rotating coaxial cylinders are solved. The results obtained may be useful in the mechanics of suspensions, magnetic and biological liquids, liquid crystals and other liquid media of complex structure. 1000 Elsevier Science Ltd. All rights reserved.
\end{abstract}

The theory proposed below is based on constitutive relations of the Cosserat continuum with a memory, i.e. an elastic medium with couple stresses, each particle of which has the degrees of freedom of an absolutely rigid body. The mechanics of elastic media with couple stresses is described in [1-9], and the constitutive relations of viscoelastic couple-stress solids can be found in [10-12]. Models of liquid media with microrotations and couple-stresses, which are called micropolar liquids, trace their origin from $[13,14]$. An extensive review of the literature on the mechanics of micropolar liquids can be found in [15]. Applications of the theory of couple-stress liquids in microfiltering and capillary flaw detection are given there. The application of asymmetrical hydrodynamics to problems of tribology are described in $[16]$.

A feature of all the models of micropolar liquids, described in [13-16], is the fact that at rest they do not differ from simple (isotropic) liquids, since the static couple stresses in them are equal to zero, while the static tensor of the force stresses is spherical. The theory given below includes the models described in [13-16] as special cases and differs essentially from these models in that, in the equilibrium state, a micropolar liquid, like a liquid crystal, possesses orientational elasticity and is capable of maintaining both the moment stresses and shear force stresses (see below, Section 2). The model of a viscoelastic micropolar liquid considered here is the most general model of an oriented liquid medium, the orientation of the particles of which is characterized by an orthonormalised triple of direction vectors. In the general case, a viscoelastic micropolar liquid can possess various memory properties with respect to the variable actual configuration.

\section{CONSTITUTIVE RELATIONS OF A MICROPOLAR LIQUID WITH A MEMORY}

We will consider the Cosserat continuum model [1-9], according to which each particle of the medium can be represented as an absolutely rigid body. Its position in space at the instant of time $t$ is given by the radius vector $\mathbf{R}(t)$, while the orientation is determined by the triple of orthonormalized vectors $\mathbf{D}_{k}(t)$ $(\mathbf{k}=1,2,3)$. In addition to the actual state we will also consider a certain initial state of the medium, in which the position and orientation of a particle are specified by a vector $\mathbf{r}$ and an orthonormalized trihedron $\mathbf{d}_{k}(k=1,2,3)$. The triples of vectors $\mathbf{D}_{k}$ and $\mathbf{d}_{k}$ generate a strictly orthogonal tensor $\mathbf{H}=$ $\mathbf{d}_{k} \otimes \mathbf{D}_{k}$, which is called the microrotation tensor [7-9].

The equations of motion of a Cosserat medium, which express the balance of momentum and angular momentum for an arbitrary particle of the body, have the form [1-9]

$$
\operatorname{Div} \mathbf{T}+\rho \mathbf{m}=\rho \frac{d \mathbf{v}}{d t}, \operatorname{Div} \mathbf{M}+\mathbf{T}_{\times}+\rho \mu=\gamma \frac{d \omega}{d t}
$$


Here $\mathbf{T}$ and $\mathbf{M}$ are the stresses and couple-stress tensors of the Cauchy type, Div is the divergence operator in Euler coordinates, $\rho$ is the density of the medium, $\mathbf{m}$ and $\boldsymbol{\mu}$ are the vectors of the mass force and mass moment, $\gamma$ is the scalar measure of rotational inertia of the particles of the continuum, $\mathbf{v}$ is the velocity vector, $\boldsymbol{\omega}$ is the vector of the angular velocity of rotation of the trihedron, $\mathbf{D}_{k}$ : $d \mathbf{D}_{k} / d t=\omega \times \mathbf{D}_{k}, d / d t$ is the material derivative with respect to time, and the symbol $\mathbf{T}_{\times}$denotes the vector invariant of the second-rank tensor $\mathbf{T}$, defined by the relation

$$
\mathbf{T}_{\times}=\left(T^{m n} \mathbf{R}_{m} \otimes \mathbf{R}_{n}\right)_{\times}=T^{m n} \mathbf{R}_{m} \times \mathbf{R}_{n}
$$

Here $\mathbf{R}_{k}$ is a certain vector basis.

Using the principle of local action [17], the constitutive relations of the Cosserat continuum with a memory in the case of finite deformations must be taken in the form

$$
\begin{aligned}
& \mathbf{T}(t)=\mathbf{A}_{1}\left[\mathbf{C}^{t}(s), \mathbf{H}^{t}(s), \operatorname{grad} \mathbf{H}^{t}(s)\right] \\
& \mathbf{M}(t)=\mathbf{A}_{2}\left[\mathbf{C}^{t}(s), \mathbf{H}^{t}(s), \operatorname{grad} \mathbf{H}^{t}(s)\right] \\
& \mathbf{C}^{t}(s)=\mathbf{C}(t-s), \mathbf{H}^{t}(s)=\mathbf{H}(t-s), \mathbf{C}(t)=\operatorname{grad} \mathbf{R}(t)(s \geqslant 0)
\end{aligned}
$$

In (1.2) $A_{1}$ and $A_{2}$ are response operators, which depend on the prehistory of the deformation gradient $\mathbf{C}^{t}(s)$ of the microrotation tensor $\mathbf{H}^{t}(s)$ and its gradient, and grad is the gradient operator in the initial state, i.e. in the reference configuration.

The requirement of invariance of constitutive relations (1.2) with respect to translations of the frame of reference of the observer is satisfied automatically, while the requirement of invariance with respect to translations of the frame of reference of the observer is satisfied automatically, while the requirement of invariance with respect to rotations will be satisfied if and only if the operators $A_{1}$ and $A_{2}$ satisfy the
condition

$$
\begin{aligned}
& A_{\alpha}\left[\mathbf{C}^{t}(s) \cdot \mathbf{Q}^{t}(s), \mathbf{H}^{t}(s) \cdot \mathbf{Q}^{t}(s), \operatorname{grad} \mathbf{H}^{t}(s) \cdot \mathbf{Q}^{t}(s)\right]= \\
& =\mathbf{Q}^{T}(t) \cdot A_{\alpha}\left[\mathbf{C}^{t}(s), \mathbf{H}^{t}(s), \operatorname{grad} \mathbf{H}^{t}(s)\right] \cdot \mathbf{Q}(t) \quad(\alpha=1,2)
\end{aligned}
$$

for any strictly orthogonal tensor $\mathbf{Q}^{(t)}(s)$. Putting $\mathbf{Q}^{t}(s)=\mathbf{H}^{t T}(s)$ in (1.3) we obtain

$$
\begin{aligned}
& \mathbf{T}(t)=\mathbf{H}^{T}(t) \cdot B_{1}\left[\mathbf{U}^{t}(s), \mathbf{L}^{\prime}(s)\right] \cdot \mathbf{H}(t) \\
& \mathbf{M}(t)=\mathbf{H}^{T}(t) \cdot B_{2}\left[\mathbf{U}^{t}(s), \mathbf{L}^{\prime}(s)\right] \cdot \mathbf{H}(t)
\end{aligned}
$$

Here $\mathbf{U}^{t}(s)$ is the prehistory of the first measure of deformation and $\mathbf{L}^{t}(s)$ is the prehistory of the flexural deformation tensor. These tensors are defined by the formulae [7-9]

$$
\mathbf{U}=\mathbf{C} \cdot \mathbf{H}^{T}, \mathbf{L} \times \mathbf{E}=-(\operatorname{grad} \mathbf{H}) \cdot \mathbf{H}^{T}
$$

where $\mathbf{E}$ is the unit tensor.

It can be seen that representations (1.4) are not only necessary but also sufficient for material independence of the frame of reference.

In addition to the tensors $U$ and $L$ we will also introduce a second measure of deformation $u$ and a measure of flexural deformation $\mathbf{K}$

$$
\mathbf{u}=\mathbf{C}^{-1} \cdot \mathbf{H}, \quad \mathbf{K}=\mathbf{C} \cdot \mathbf{B} \cdot \mathbf{H}^{T}=\mathbf{L}+\mathbf{b}
$$

In (1.6) we have denoted by $\mathbf{b}$ and $\mathbf{B}$ the microstructure curvature tensors in the reference and actual configurations, respectively

$$
\mathbf{b}=-1 / 2\left(\operatorname{grad} \mathbf{d}_{k}\right) \times \mathbf{d}_{k}, \quad \mathbf{B}=-1 / 2\left(\operatorname{Grad} \mathbf{D}_{k}\right) \times \mathbf{D}_{k}
$$

where Grad is the gradient operator in Euler coordinates.

Note that the quantities $\mathbf{b}, \mathbf{B}, \mathbf{K}, \mathbf{L}$ and $\mathbf{M}$ are not true, but second-rank axial tensors (pseudotensors). This must be taken into account when formulating the properties of isotropy of the tensor functions or operators in which these tensors participate [6].

The operators $B_{1}$ and $B_{2}$ in constitutive relations (1.4), generally speaking, depend on certain constant tensors (i.e. which do not change during deformation), defined by the choice of reference 
configuration of the material body. The tensor of the curvature of the initial state $b$, in particular, is such a tensor. Hence, taking (1.6) into account, constitutive relations (1.4) can be represented in the form

$$
\begin{aligned}
& \mathbf{T}(t)=\mathbf{H}^{T}(t) \cdot D_{1}\left[\mathbf{U}^{\prime}(s), \mathbf{K}^{\prime}(s)\right] \cdot \mathbf{H}(t) \\
& \mathbf{M}(t)=\mathbf{H}^{T}(t) \cdot D_{2}\left[\mathbf{U}^{\prime}(s), \mathbf{K}^{\prime}(s)\right] \cdot \mathbf{H}(t)
\end{aligned}
$$

In the case of an elastic material the tensors $\mathbf{T}$ and $\mathbf{M}$ are independent of the deformation prehistory, and constitutive relations (1.8) take the form

$$
\begin{aligned}
& \mathbf{T}(t)=\mathbf{H}^{T}(t) \cdot f_{1}[\mathbf{U}(t), \mathbf{K}(t)] \cdot \mathbf{H}(t) \\
& \mathbf{M}(t)=\mathbf{H}^{T}(t) \cdot f_{2}[\mathbf{U}(t), \mathbf{K}(t)] \cdot \mathbf{H}(t)
\end{aligned}
$$

where $f_{1}$ and $f_{2}$ are tensor functions. Using the laws of thermodynamics the equations of state (1.9) of the Cosserat elastic continuum can be represented using the specific deformation potential energy $W_{0}(\mathbf{U}, \mathbf{K})$, which is identical with the specific free energy in an isothermal process and the specific internal energy in an adiabatic process, as follows [9]:

$$
\begin{aligned}
& \mathbf{T}=\rho \mathbf{C}^{T} \cdot(\partial W / \partial \mathbf{U}) \cdot \mathbf{H}, W=\rho_{0}^{-1} W_{0} \\
& \mathbf{M}=\rho \mathbf{C}^{T} \cdot(\partial W / \partial \mathbf{K}) \cdot \mathbf{H}
\end{aligned}
$$

Here $W$ is the mass deformation potential energy density and $\rho_{0}$ is the density of the material in the reference configuration.

Henceforth we will consider a class of isotropic materials, for which the tensor response operators $D_{1}$ and $D_{2}$ in (1.8) are isotropic, i.e. they satisfy the relations $(\eta=\operatorname{det} \mathbf{Q})$

$$
\begin{aligned}
& D_{1}\left[\mathbf{Q} \cdot \mathbf{U}^{\prime}(s) \cdot \mathbf{Q}^{T}, \eta \mathbf{Q} \cdot \mathbf{K}^{\prime}(s) \cdot \mathbf{Q}^{T}\right]=\mathbf{Q} \cdot D_{1}\left[\mathbf{U}^{\prime}(s), \mathbf{K}^{\prime}(s)\right] \cdot \mathbf{Q}^{T} \\
& \eta D_{2}\left[\mathbf{Q} \cdot \mathbf{U}^{\prime}(s) \cdot \mathbf{Q}^{T}, \eta \mathbf{Q} \cdot \mathbf{K}^{\prime}(s) \cdot \mathbf{Q}^{T}\right]=\mathbf{Q} \cdot D_{2}\left[\mathbf{U}^{\prime}(s), \mathbf{K}^{\prime}(s)\right] \cdot \mathbf{Q}^{T}
\end{aligned}
$$

for any orthogonal tensor $\mathbf{Q}$. According to (1.5), (1.8) and (1.11) the tensors $\mathbf{T}(t)$ and $\mathbf{M}(t)$ are not changed when the following replacements are made

$$
\mathbf{C}^{\prime}(s) \rightarrow \mathbf{Q} \cdot \mathbf{C}^{\prime}(s), \quad \mathbf{H}^{\prime}(s) \rightarrow \mathbf{Q} \cdot \mathbf{H}^{\prime}(s), \quad \forall \mathbf{Q}=\mathbf{Q}^{-T}
$$

This means that isotropic materials are insensitive to any changes in the reference configuration, described by orthogonal transformations.

In the mechanics of simple materials with a memory, the idea of the relative deformation gradient $C_{t}(\tau)=\mathbf{C}^{-1}(t) \cdot \mathbf{C}(\tau)$ is used $[17,18]$, when determining which the actual configuration is considered as the reference configuration, and the configuration corresponding to the instant of time $\tau$, plays the role of the actual configuration. In a similar way we introduce the idea of the relative microrotation

$$
\mathbf{H}_{r}(\tau)=\mathbf{D}_{k}(t) \otimes \mathbf{D}_{k}(\tau)=\mathbf{H}^{T}(t) \cdot \mathbf{H}(\tau)
$$

and also the relative measures of deformation

$$
\begin{aligned}
& \mathbf{U}_{t}(\tau)=\mathbf{C}_{t}(\tau) \cdot \mathbf{H}_{t}^{T}(\tau), \quad \mathbf{K}_{t}(\tau)=\mathbf{L}_{t}(\tau)+\mathbf{B}(t) \\
& \mathbf{L}_{t}(\tau) \times \mathbf{E}=-\left[\operatorname{Grad} \mathbf{H}_{t}(\tau)\right] \cdot \mathbf{H}_{t}^{T}(\tau)
\end{aligned}
$$

The prehistories of tensors (1.13) and (1.14) are denoted as follows:

$$
\mathbf{C}_{t}(t-s) \equiv \mathbf{C}_{t}^{t}(s), \quad \mathbf{H}_{t}(t-s) \equiv \mathbf{H}_{t}^{t}(s) \text { etc. }
$$

The following formulae are obtained from (1.13) and (1.14)

$$
\begin{aligned}
& \mathbf{U}^{t}(s)=\mathbf{U}(t) \cdot \mathbf{H}(t) \cdot \mathbf{U}_{t}^{t}(s) \cdot \mathbf{H}^{T}(t) \\
& \mathbf{K}^{t}(s)=\mathbf{U}(t) \cdot \mathbf{H}(t) \cdot \mathbf{K}_{t}^{t}(s) \cdot \mathbf{H}^{T}(t) \\
& \mathbf{U}_{t}^{t}(0)=\mathbf{E}, \quad \mathbf{K}_{t}^{t}(0)=\mathbf{B}(t), \quad \mathbf{L}_{t}^{t}(0)=0 \\
& \mathbf{K}_{t}^{t}(s)=\mathbf{L}_{t}^{t}(s)+\mathbf{B}(t)
\end{aligned}
$$


Theorem 1. Constitutive relations of any isotropic Cosserat medium with a memory can be represented in the form

$$
\mathbf{T}(t)=F_{1}\left[\mathbf{u}(t), \mathbf{U}_{t}^{t}(s), \mathbf{K}_{t}^{t}(s)\right], \quad \mathbf{M}(t)=F_{2}\left[\mathbf{u}(t), \mathbf{U}_{t}^{t}(s), \mathbf{K}_{t}^{t}(s)\right]
$$

where $F_{1}$ and $F_{2}$ are isotropic operators.

Proof. On the basis of (1.15) constitutive relation (1.8) for the stress tensor can be represented in the form

$$
\mathbf{T}(t)=\mathbf{H}^{T}(t) \cdot G_{\mathrm{l}}\left[\mathbf{U}(t), \mathbf{H}(t) \cdot \mathbf{U}_{t}^{t}(s) \cdot \mathbf{H}^{T}(t), \mathbf{H}(t) \cdot \mathbf{K}_{t}^{t}(s) \cdot \mathbf{H}^{T}(t)\right] \cdot \mathbf{H}(t)
$$

We put $\mathbf{Q}=\mathbf{H}^{T}(t)$ in (1.12). Then the tensor $\mathrm{U}(t)$, by (1.6), is replaced by a tensor $\mathbf{u}^{-1}(t)$, while the tensor $\mathbf{H}(t)$ is replaced by the unit tensor $\mathrm{E}$, which, on the basis of (1.18), gives

$$
\mathbf{T}(t)=G_{1}\left[\mathbf{u}^{-1}(t), \mathbf{U}_{t}^{t}(s), \mathbf{K}_{t}^{t}(s)\right]
$$

The constitutive relation for the couple-stress tensor $\mathbf{M}(t)$ is converted in a similar way and leads to expressions (1.17). The isotropic nature of the operators $F_{1}$ and $F_{2}$ follows from the property of isotropy of the operators $D_{1}$ and $D_{2}$.

A Cosserat medium with a memory which is insensitive to any changes in the reference configuration, and which preserves the density of the medium, will be called a viscoelastic micropolar liquid. It is obvious that the liquid belongs to the class of isotropic materials. Since the tensors $\mathbf{U}_{t}^{t}(s)$ and $\mathbf{K}_{t}^{t}(s)$ in $(1.17)$ are independent of the choice of the reference configuration, an isotropic medium will be a liquid if and only if the dependence on the tensor $\mathbf{u}(t)$ in the constitutive relations reduces to a dependence on $\operatorname{det} \mathbf{u}(t)$ or, which is equivalent, to a dependence on the density $\rho(t)$. Hence the following theorem holds.

Theorem 2. The general representation of the constitutive equations of a viscoelastic micropolar liquid has the form

$$
\begin{gathered}
\mathbf{T}(t)=H_{1}\left[\rho(t), \mathbf{B}(t), \mathbf{U}_{t}^{t}(s), \mathbf{L}_{t}^{t}(s)\right] \\
\mathbf{M}(t)=H_{2}\left[\rho(t), \mathbf{B}(t), \mathbf{U}_{t}^{t}(s), \mathbf{L}_{t}^{t}(s)\right]
\end{gathered}
$$

where $H_{1}$ and $H_{2}$ are isotropic operators.

Relation (1.16) has been taken into account in (1.19).

Theorem 2 is an extension of Noll's theorem [17] to simple liquids in the case of a micropolar liquid.

For a liquid at rest we have $\mathbf{L}_{t}^{t}(s)=0, \mathbf{U}_{t}^{t}(s)=\mathbf{E}$, and constitutive relations (1.19) take the form of the equations of state of an elastic micropolar liquid

$$
\mathbf{T}=\varphi(\rho, \mathbf{B}), \quad \mathbf{M}=\psi(\rho, \mathbf{B})
$$

where $\varphi$ and $\psi$ are isotropic tensor functions.

The model of an elastic micropolar liquid can be obtained in a different way, namely, as a special case of an elastic isotropic Cosserat medium, for which the functions $f_{1}$ and $f_{2}$ in (1.9) are isotropic. Assuming $\mathbf{Q}=\mathbf{H}^{T}$ in the isotropicity condition, we obtain from (1.9) a representation of the equations of state of an isotropic elastic
material

$$
\begin{aligned}
& \mathbf{T}=f_{1}\left(\mathbf{u}^{-1}, \mathbf{u}^{-1} \cdot \mathbf{B}\right)=\varphi(\mathbf{u}, \mathbf{B}) \\
& \mathbf{M}=f_{2}\left(\mathbf{u}^{-1}, \mathbf{u}^{-1} \cdot \mathbf{B}\right)=\psi(\mathbf{u}, \mathbf{B})
\end{aligned}
$$

In relations (1.21) only the first tensor argument of the isotropic functions $\varphi$ and $\psi$ depends on the choice of the reference configuration. The property of insensitivity of the material to any changes in the reference configuration, with the condition that the volume is preserved, will be satisfied if and only if the dependence on $\mathbf{u}$ in (1.21) reduces to a dependence on det $\mathbf{u}$. This leads to constitutive relations $(1.20)$.

Relations (1.19) contain a model of a micropolar liquid of the differential type. As we know [8], the rates of deformation and flexural deformation tensors are defined by the formulae 


$$
\begin{aligned}
& \mathbf{\epsilon} \equiv \mathbf{C}^{-1} \cdot\left(\frac{d}{d t} \mathbf{U}\right) \cdot \mathbf{H}=\operatorname{Grad} \mathbf{v}+\mathbf{E} \times \boldsymbol{\omega} \\
& \boldsymbol{x} \equiv \mathbf{C}^{-1} \cdot\left(\frac{d}{d t} \mathbf{K}\right) \cdot \mathbf{H}=\operatorname{Grad} \boldsymbol{\omega}
\end{aligned}
$$

We will introduce indifferent rate tensors of higher order by the recursion relations

$$
\begin{aligned}
& \mathbf{A}_{n+1}=\frac{d}{d t} \mathbf{A}_{n}+(\operatorname{Grad} \mathbf{v}) \cdot \mathbf{A}_{n}+\mathbf{A}_{n} \times \boldsymbol{\omega}, \quad \mathbf{A}_{0}=\mathbf{E}, \quad \mathbf{A}_{\mathbf{l}}=\mathbf{\epsilon} \\
& \mathbf{B}_{n+1}=\frac{d}{d t} \mathbf{B}_{n}+(\mathrm{Grad} \mathbf{v}) \cdot \mathbf{B}_{n}+\mathbf{B}_{n} \times \omega, \quad \mathbf{B}_{0}=\mathbf{B}, \quad \mathbf{B}_{1}=x
\end{aligned}
$$

Taking into account the formal expansions

$$
\mathbf{U}_{t}^{t}(s)=\sum_{n=0}^{\infty} \frac{(-1)^{n}}{n !} s^{n} \mathbf{A}_{n}(t), \quad \mathbf{L}_{t}^{t}(s)=\sum_{n=1}^{\infty} \frac{(-1)^{n}}{n !} s^{n} \mathbf{B}_{n}(t)
$$

we will call a micropolar liquid with the equations of state

$$
\begin{aligned}
& \mathbf{T}=f_{1}\left(\rho, \mathbf{B}, \mathbf{A}_{1} \ldots \mathbf{A}_{m}, \mathbf{B}_{1} \ldots \mathbf{B}_{n}\right) \\
& \mathbf{M}=f_{2}\left(\rho, \mathbf{B}, \mathbf{A}_{1} \ldots \mathbf{A}_{m}, \mathbf{B}_{1} \ldots \mathbf{B}_{n}\right)
\end{aligned}
$$

$\left(f_{1}\right.$ and $f_{2}$ are isotropic functions), a differential-type liquid of $(m, n)$ complexity.

A special case of $(1.24)$ is a model of a viscous micropolar liquid [13,14], the equations of state of which have the form

$$
\mathbf{T}=f_{1}(\rho, \epsilon), \quad \mathbf{M}=f_{2}(\rho, \boldsymbol{x})
$$

\section{THE EQUATIONS OF AN ELASTIC MICROPOLAR LIQUID}

For an elastic liquid in an isothermal process, the mass free energy density $W$ is an isotropic function of the curvature tensor $\mathbf{B}$ and the density $\rho$. Taking into account the relation

$$
\frac{d}{d t} \mathbf{B}=x-\mathbf{E} \cdot \mathbf{B}+\boldsymbol{\omega} \times \mathbf{B}-\mathbf{B} \times \omega
$$

which follows from (1.7) and (1.22), we calculate the rate of change of the function $W$ in a fixed particle of the medium

$$
\begin{aligned}
& \frac{d W}{d t}=\operatorname{tr}\left(\frac{\partial W}{\partial \mathbf{B}} \cdot \frac{d \mathbf{B}^{T}}{d t}\right)+\frac{\partial W}{\partial \mathbf{p}} \frac{d \rho}{d t}=\operatorname{tr}\left(\frac{\partial W}{\partial \mathbf{B}} \cdot x^{T}\right)-\operatorname{tr}\left[\left(\frac{\partial W}{\partial \mathbf{B}} \cdot \mathbf{B}^{T}+\rho \frac{\partial W}{\partial p} \mathbf{E}\right) \cdot \mathbf{\epsilon}^{T}\right]+ \\
& +\operatorname{tr}\left[\left(\mathbf{B}^{T} \cdot \frac{\partial W}{\partial \mathbf{B}}-\frac{\partial W}{\partial \mathbf{B}} \cdot \mathbf{B}^{T}\right) \times \omega\right]
\end{aligned}
$$

On the other hand, from (1.10) and (1.22) we have

$$
\frac{d W}{d t}=\rho^{-1} \operatorname{tr}\left(\mathbf{T} \cdot \epsilon^{T}+\mathbf{M} \cdot x^{T}\right)
$$

Comparing (2.2) and (2.3) and taking into account the fact that the tensors $\boldsymbol{\epsilon}, \boldsymbol{x}$ for the motion of the medium can take arbitrary values, we obtain

$$
\mathbf{M}=\rho \frac{\partial W}{\partial \mathbf{B}}, \quad \mathbf{T}=-p \mathbf{E}-\mathbf{M} \cdot \mathbf{B}^{T}, \quad p=\rho^{2} \frac{\partial W}{\partial \rho}
$$

The following relation is obtained from the fact that the vector $\omega$ is arbitrary and using (2.2)-(2.4) 


$$
\left(\mathbf{M} \cdot \mathbf{B}^{T}\right)_{\times}=\left(\mathbf{B}^{T} \cdot \mathbf{M}\right)_{\times}
$$

It can be shown that the equations of state (2.4) of an elastic liquid hold for any thermodynamic processes, where the free energy $W$ must also be assumed to depend on the temperature.

The model of an elastic micropolar liquid is similar to the model of a nematic liquid crystal [19-23], but differs from it in the fact that the orientation of the particles of the micropolar liquid is specified by an orthonormalized triple of vectors, whereas in the continuum theory of nematic liquid crystals the orientation is characterized by a single unit vector - the director $\mathbf{n}$. The free energy $W$ in the theory of nematic liquid crystals is an isotropic function of two arguments [19-21]: the vector $\mathbf{n}$ and the tensor Grad $\mathbf{n}$, whereas in a micropolar liquid $W$ depends on one tensor argument $\mathbf{B}$. Of course, in both cases, there are also scalar arguments: the density and the temperature.

Note that the property of isotropicity of the function $W(\rho, \mathbf{B})$ does not mean that the elastic micropolar liquid is an isotropic liquid. The last term is equivalent to the notion of a simple elastic liquid and corresponds to the case when $W=W \rho$ ). In just the same way the property of isotropicity of the free-energy function of nematic liquid crystal $W(\rho, \mathbf{n}, \operatorname{Grad} \mathbf{n})$ does not prevent us calling it an isotropic liquid [24].

The equations of state of an elastic micropolar liquid (2.4) can be used to model the behaviour of complex microstructural liquids, similar to liquid crystals. The anisotropy of the properties and the orientational elasticity of nematic liquid crystals can be explained by the considerable preferential orientation of the elongated molecules or the complexes generated by them, where the microstructure of the liquid crystal can be represented by floating long rods or filaments. By analogy with this representation we can regard the microstructure of the elastic micropolar liquid (2.4) as formed by an ordered set of floating ellipsoids with different semiaxes.

The complete system of equations of motion of elastic micropolar liquids in Euler coordinates contains, as the unknown functions, the density $\rho$, the velocity field $\mathbf{v}$, the angular velocity field $\omega$, and the tensor curvature field $\mathbf{B}$, and according to (1.1), (1.22) and (2.4) has the form

$$
\begin{aligned}
& -\operatorname{Grad} p-\operatorname{Div}\left(\mathbf{M} \cdot \mathbf{B}^{T}\right)+\rho \mathbf{m}=\rho\left(\frac{\partial \mathbf{v}}{\partial t}+\mathbf{v} \cdot \operatorname{Grad} \mathbf{v}\right) \\
& \operatorname{Div} \mathbf{M}-\left(\mathbf{M} \cdot \mathbf{B}^{T}\right)_{\times}+\rho \boldsymbol{\mu}=\gamma\left(\frac{\partial \omega}{\partial t}+\mathbf{v} \cdot \operatorname{Grad} \omega\right) \\
& \frac{\partial \mathbf{B}}{\partial t}+\mathbf{v} \cdot \operatorname{Grad} \mathbf{B}=\operatorname{Grad} \omega-(\operatorname{Grad} \mathbf{v}) \cdot \mathbf{B}-\mathbf{B} \times \omega \\
& \frac{\partial \rho}{\partial t}+\operatorname{Div}(\rho \mathbf{v})=0
\end{aligned}
$$

The quantities $p$ and $\mathbf{M}$ are assumed to be expressible in terms of $\rho$ and B using the equations of state (2.4). The rotational inertia $\gamma$, generally speaking, can be specified by a function of the density $\rho$. In the case of an incompressible liquid $\rho=$ const, and the pressure $p$ cannot be expressed in terms of $\rho$ and $\mathbf{B}$ and is an unknown function of the coordinates and time.

In the problem of a liquid at rest $\mathbf{v}=\boldsymbol{\omega}=0$, and Eqs (2.6) become the equilibrium equations. The following identity follows from the homogeneity of the liquid and relations (2.4) and (2.5)

$$
\operatorname{Grad}\left(W+\rho^{-1} p\right)=\rho^{-1}\left[\operatorname{Grad} p+\operatorname{Div}\left(\mathbf{M} \cdot \mathbf{B}^{T}\right)\right]-\rho^{-1}\left[\operatorname{Div} \mathbf{M}-\left(\mathbf{M} \cdot \mathbf{B}^{T}\right)_{\times}\right] \cdot \mathbf{B}^{T}
$$

When $\boldsymbol{\mu}=0$, i.e. when there are no external mass moments, the force equilibrium equation, according to $(2.6)$ and $(2.7)$, reduces to the form

$$
\operatorname{Grad}\left(W+\rho \frac{\partial W}{\partial \rho}\right)=\mathbf{m}
$$

and will be solvable in the case of potential mass forces $\mathbf{m}$. For an incompressible liquid the expression in parenthesis in (2.8) must be replaced by $W+\rho^{-1} p$. Hence, the determination of the equilibrium state of an elastic micropolar liquid reduces to determining the orthonormalized triple of vectors $\mathbf{D}_{k}$ from the equations

$$
\operatorname{Div} \mathbf{M}-\left(\mathbf{M} \cdot \mathbf{B}^{T}\right)_{\times}=0, \quad \mathbf{M}=\rho \frac{\partial W}{\partial \mathbf{B}}, \quad \mathbf{B}=-1 / 2\left(\operatorname{Grad} \mathbf{D}_{k}\right) \times \mathbf{D}_{k}
$$


and then finding the density or pressure using (2.8).

Since, by (2.4), the Cauchy stress tensor in an elastic liquid is not spherical, a micropolar liquid at rest is capable of withstanding shear force stresses and also couple stresses.

When considering the problem of the equilibrium of a micropolar liquid, it is necessary to add boundary conditions on the boundary $\Sigma$ of the volume $V$, occupied by the liquid, to Eqs (2.9). These conditions consist in specifying the trihedron $\mathbf{D}_{k}$ or the vector of the couple-stress load: $\mathbf{N} \cdot \mathbf{M}=\mathbf{1}$, where $\mathbf{N}$ is the unit normal to the boundary. It can be verified that this boundary-value problem, in the case of an incompressible liquid, can be formulated in the form of a variational principle

$$
\begin{aligned}
& \delta \iiint_{V} \rho W d V-\iint_{\Sigma_{2}} 1 \cdot \psi d \Sigma=0 \\
& \left.\psi\right|_{\Sigma_{1}}=0, \quad \Sigma=\Sigma_{1} \cup \Sigma_{2}, \quad \psi=-1 / 2 \delta D_{k} \times D_{k}
\end{aligned}
$$

Here $\Sigma_{1}$ is part of the surface $\Sigma$ on which the trihedron $\mathbf{D}_{k}$ is specified, $\Sigma_{2}$ is the part of the surface on which the couple-stress load is specified and $\psi$ is the virtual orientation vector, in terms of which the variation of the curvature tensor is expressed

$$
\delta B=\operatorname{Grad} \psi-B \times \psi
$$

We can eliminate the vectors $D_{k}$ from the system of equations (2.9) by replacing the last relation in (2.9) by the equation of compatibility with respect to the tensor B (Rot is the curl operator in Euler coordinates)

$$
(\operatorname{Rot} B)^{T}=\mathbf{B}^{2}-(\operatorname{tr} B) B+1 / 2\left(\operatorname{tr}^{2} B-\operatorname{tr} B^{2}\right) \mathbf{E}
$$

Using the Hamilton-Cayley formula for the non-singular tensor B, Eq. (2.12) can be written differently

$$
(\operatorname{RotB})^{T}=\mathbf{B}^{-1} \operatorname{detB}
$$

In the simply connected region, the triple of orthonormalized vectors $\mathbf{D}_{k}$ is determined from the specified smooth tensor field B, which satisfies compatibility condition (2.12), uniquely, if the trihedron $\mathbf{D}_{k}$ is specified at a certain point of the region.

Since the curvature of the microstructure $\mathbf{B}$ is a pseudotensor of the second rank, the free energy $W$ is an even function of $\mathbf{B}: W(\rho-\mathbf{B})=W(\rho, \mathbf{B})$. The simplest example of an even function is the quadratic form, the general representation of which, taking into account the isotropicity of the function $W(\mathbf{B})$, in the case of an incompressible medium has the form

$$
\rho W=1 / 2\left[\lambda \operatorname{tr}^{2} \mathbf{B}+\mu \operatorname{tr}\left(\mathbf{B} \cdot \mathbf{B}^{T}\right)+v \operatorname{tr} \mathbf{B}^{2}\right]
$$

where $\lambda, \mu$ and $v$ are constants.

It can be shown that the necessary and sufficient conditions for the form (2.14) to be positive definite is the satisfaction of the inequalities

$$
3 \lambda+\mu+v>0, \mu+v>0, \mu>0
$$

According to (2.4) the following linear dependence of the couple-stress tensor on the curvature of the structure corresponds to expression (2.14)

$$
\mathbf{M}=\lambda \mathbf{E t r B}+\mu \mathbf{B}+v B^{T}
$$

As in the non-linear theory of elasticity [17] it is convenient to formulate additional limitations on the form of the relationship $W(\mathbf{B})$ in addition to it being positive definite. In particular, this condition can be the condition for strong ellipticity of the equilibrium equations. It can be shown that the condition of strong ellipticity of the couple-stress equilibrium equation (2.9) is equivalent to the inequality

$$
\left.\frac{d^{2}}{d \tau^{2}} W(\mathbf{B}+\tau c \otimes d)\right|_{\tau=0}>0
$$

for any non-zero vectors $\mathbf{c}$ and $\mathbf{d}$. 
For the equation of state (2.14), condition (2.17) is satisfied if and only if

$$
\mu>0, \mu+v+\lambda>0
$$

As in the case of the linear theory of elasticity, the last inequalities follow from the conditions for (2.15) to be positive definite. Nevertheless, for equations of state of general form, condition (2.17) does not follow from the fact that the function $W(\mathbf{B})$ is positive.

\section{SOME PROBLEMS ON THE EQUILIBRIUM OF ELASTIC LIQUIDS}

We will henceforth confine ourselves to finding the equilibrium solutions in the case of homogeneous incompressible liquid when there are no volume moments.

$A$ twisted structure. Consider the following field of director vectors

$$
\begin{aligned}
& \mathbf{D}_{1}=\mathbf{e}_{1} \cos \alpha(Z)+\mathbf{e}_{2} \sin \alpha(Z) \\
& \mathbf{D}_{2}=-\mathbf{e}_{1} \sin \alpha(Z)+\mathbf{e}_{2} \cos \alpha(Z), \quad \mathbf{D}_{3}=\mathbf{e}_{3}
\end{aligned}
$$

Here and henceforth $\mathbf{e}_{1}, \mathbf{e}_{2}$ and $\mathbf{e}_{3}$ are an orthonormalized Cartesian basis, and $X, Y$ and $Z$ are Cartesian coordinates.

The curvature tensor $\mathbf{B}$, corresponding to (3.1), is defined by the relation $\mathbf{B}=\alpha^{\prime}(Z) \mathbf{e}_{3} \otimes \mathbf{e}_{3}$. It follows from the property of isotropicity of the function $\mathbf{M}(\mathbf{B})$ that $\left(\mathbf{M} \cdot \mathbf{B}^{T}\right)_{\times}=0$ for arbitrary constitutive relations. Assuming that $\alpha^{\prime}=$ const, the tensor $\mathbf{M}$ is constant and Eq. (2.9) is satisfied identically. Hence, the structure of (3.1) when $\alpha(Z)=a Z+b$ is a universal solution which is independent of the choice of the equations of state.

The plane problem. In this case the orientation of the particles of the micropolar liquid is defined by a single parameter - the angle of rotation $\alpha(X, Y)$ of the trihedron $\mathbf{D}_{k}$ around a certain axis. To fix our ideas we will assume that the axis coincides with the direction of $\mathbf{D}_{3}$. Then the trihedron $\mathbf{D}_{k}$ is defined by the following equations

$$
\begin{aligned}
& \mathbf{D}_{1}=\mathbf{e}_{1} \cos \alpha(X, Y)+\mathbf{e}_{2} \sin \alpha(X, Y) \\
& \mathbf{D}_{2}=-\mathbf{e}_{1} \sin \alpha(X, Y)+\mathbf{e}_{2} \cos \alpha(X, Y), \quad \mathbf{D}_{3}=\mathbf{e}_{3}
\end{aligned}
$$

For (3.2) the curvature tensor $B$ is given by the formula

$$
\mathbf{B}=\mathbf{e}_{1} \otimes \mathbf{e}_{3} \frac{\partial \alpha}{\partial X}+\mathbf{e}_{2} \otimes \mathbf{e}_{3} \frac{\partial \alpha}{\partial Y}=(\operatorname{Grad} \alpha) \otimes \mathbf{e}_{3}
$$

It follows from (2.4), (3.2) and (3.3) that for the deformation (3.2) all the components of $\mathbf{M}$ vanish, apart from $M_{13}, M_{23}, M_{31}$ and $M_{32}$. In addition, the equation $\left(\mathbf{M} \cdot \mathbf{B}^{T}\right)_{\times}=0$ is satisfied. Hence, of the three moment equations of equilibrium (2.9) two are satisfied identically, while the last takes the form

$$
\frac{\partial M_{13}}{\partial X}+\frac{\partial M_{23}}{\partial Y}=0
$$

An example of plane deformation is a curved structure, defined by the relations

$$
\begin{aligned}
& \mathbf{D}_{1}=\mathbf{e}_{R} \cos \beta \Phi+\mathbf{e}_{\Phi} \sin \beta \Phi, \quad \beta=\text { const } \\
& \mathbf{D}_{2}=-\mathbf{e}_{R} \sin \beta \Phi+\mathbf{e}_{\boldsymbol{\Phi}} \cos \beta \Phi, \quad \mathbf{D}_{3}=\mathbf{e}_{Z}
\end{aligned}
$$

Here $R, \Phi$ and $Z$ are polar coordinates and $\mathbf{e}_{R}, \mathbf{e}_{\Phi}$ and $\mathbf{e}_{z}$ are coordinate unit vectors. The curvature tensor $B$ is given by the formula

$$
\mathbf{B}=\frac{1+\beta}{R} \mathbf{e}_{\Phi} \otimes \mathbf{e}_{Z}
$$

It can be shown that solution (3.5) is universal, like solution (3.1).

Consider the plane problem for constitutive relations (2.14). Taking (3.3) into account, we write equation of state (2.16) in the form 


$$
M=\mu(\operatorname{Grad} \alpha) \otimes e_{3}+v e_{3} \otimes(G r a d \alpha)
$$

while equilibrium equation (3.4) is reduced to Laplace's equation

$$
\mu \Delta \alpha=0
$$

Hence, for the law of state (2.14) the determination of the structure of a micropolar liquid in the plane problem reduces to finding a harmonic function. Note that the same problem is obtained when investigating the plane deformation of a nematic liquid crystal in the single-constant approximation.

Equilibrium micropolar liquids with a free surface. Consider the problem of the equilibrium of a heavy incompressible liquid having a free surface and contained in an infinitely long cylindrical vessel. We will write the equation of the free surface $\Sigma$ in the form

$$
Z=\zeta(X, Y),(X, Y) \in \Omega
$$

where the region $\Omega$ is the cross-section of the cylinder. The free boundary $\Omega$ possesses surface energy, which we will calculate from the formula

$$
\pi=\int_{\Sigma} \sigma d \Sigma
$$

Here $\sigma$ is the constant surface tension.

We will derive the boundary conditions on $\Sigma$, as in the case of a simple liquid, by a variational method [25]. By varying the total energy functional, assuming that there are no external loads on the surface $\Sigma$, we obtain the following equation for the function

$$
\nabla \cdot\left(\frac{1}{\sqrt{1+|\nabla \zeta|^{2}}} \nabla \zeta\right)=h \zeta+\left.\frac{1}{\sigma} W\right|_{Z=\zeta(X, Y)}(X, Y) \in \Omega
$$

In (3.7) $\nabla$ is the gradient operator in the $(X, Y)$ plane, $g$ is the acceleration due to gravity and $h=$ $\rho g / \sigma$ is the capillary constant. The last term in (3.7) distinguishes the relation obtained from the equations of the capillary surface of a simple liquid [25].

The following conditions, which consist of specifying the angle of contact $\xi$ of the free surface $\Sigma$ with the vessel walls, are satisfied on the boundary of the region $\Omega$

$$
\mathbf{N} \cdot \frac{1}{\sqrt{1+|\nabla \zeta|^{2}}} \nabla \zeta=\cos \xi
$$

( $\mathbf{N}$ is the outward unit normal to the cylinder wall).

Hence, the problem of determining the form of the capillary surface of the micropolar liquid reduces to equilibrium equations (2.8) and (2.9), the boundary conditions on the cylinder surface and relations (3.7) and (3.8).

Equations (3.7) and (3.8) enable us to draw a qualitative conclusion regarding the influence of the microstructure on the volume of the liquid elevated above a certain initial level in the vessel due to the action of capillary effects. Namely, suppose $V_{0}$ is the volume specified above in the case of a simple liquid, and $V$ is the volume of the elevated micropolar liquid in the same vessel. Then, integrating Eq. (3.7) taking boundary condition (3.8) into account, we obtain that

$$
V-V_{0}=-\left.\int_{\Omega} \frac{1}{\rho g} W\right|_{Z=\zeta(X, Y)} d \Omega, \quad V_{0}=\frac{1}{h} \int_{\partial \Omega} \cos \xi d s
$$

Since the free energy density $W$ is negative, the last relation shows that, in the case of a micropolar liquid, the volume of the elevated liquid will be less.

Equilibrium of the phases of a micropolar liquid. Consider the problem of the equilibrium of the phases of micropolar liquid in a uniform temperature field. The conditions for thermodynamic equilibrium of the phases of an elastic material with couple stresses of general form were obtained in [26]. For a micropolar liquid the conditions for phase equilibrium can be obtained directly using variational equation 
(2.10), taking into account the fact that the interface between the phases may vary independently of the field of the director vectors $\mathbf{D}_{k}$.

For a microcoherent phase transition, i.e. in the case of a field $\mathbf{D}_{k}$ that is continuous in the neighbourhood of the interface, the jumps in the virtual orientation vector $\psi$ and the curvature tensor $\mathbf{B}$ are related by the equation

$$
[\boldsymbol{\psi}]_{-}^{+}+c \mathbf{N} \cdot[\mathbf{B}]_{-}^{+}=0
$$

In (3.9) $c$ is the velocity of motion of the interface in the direction of the normal, and the square brackets denote a jump in the corresponding value on crossing the interface. For a phase transition with microslip at the interface the vector $\mathbf{N} \cdot \mathbf{M}$ vanishes.

The following additional boundary condition on the phase boundary, necessary to determine it, follows from stationarity condition (2.10) for microcoherent phase transitions and phase transitions with microslip

$$
[W+p / \rho]_{-}^{+}=0
$$

It can be shown that Eq. (3.10) also follows from the condition for thermodynamic equilibrium [26] using equations of state (2.4).

As an example we will consider the problem of the formation of a new phase-a simple incompressible liquid in the neighbourhood of curved structure (3.5). In view of the symmetry of solution (3.5) the region occupied by the new phase is a circular cylinder of radius $\delta$. We will assume that the densities of the phases are identical and equal to $\rho$. We will choose the equation of state for the micropolar liquid in the form (2.14), and for a simple liquid the mass free energy density $W_{-}$is constant. Then, the radius of a phase inclusion is found from Eq. (3.10) and is given by the formula

$$
\delta=(1+\beta) \sqrt{\mu /\left(2 \rho W_{-}\right)}
$$

\section{THE VISCOMETER FLOWS OF AN INCOMPRESSIBLE MICROPOLAR LIQUID}

Viscometer flows occupy an important place in the theory of simple Newtonian liquids [17, 18]. In this type of flow an arbitrary Newtonian liquid behaves as a differential-type liquid. Such flows play a considerable role in experimental investigations of the properties of viscoelastic liquids, and enables one, in particular, to determine the constants that occur in the equations of state.

Shear flow. The simplest example of viscometer flow is the plane steady flow of a liquid in a layer of depth $h(-\infty<X<\infty, 0 \leqslant Y \leqslant h,-\infty<Z<\infty)$ due either to shear stresses $\left(T_{21}=\tau\right)$ applied to the surface $Y=h$, or by specifying a shear velocity $\left(v=v^{0} e_{1}\right)$ (linear Couette flow [18]).

We will consider the plane motion of a micropolar liquid in more detail. In such a liquid the director vectors $\mathbf{D}_{k}$ are defined by formulae (3.2), taking into account the fact that the angle of rotation $\alpha$ also depends on the time: $\alpha=\alpha(X, Y, t)$. The velocity and angular velocity fields have the form

$$
\mathbf{v}=v_{1}(X, Y, t) \mathbf{e}_{1}+v_{2}(X, Y, t) \mathbf{e}_{2}, \omega=\omega(X, Y, t) \mathbf{e}_{3}
$$

The angular velocity $\omega$ is related to the angle of rotation by the formula

$$
\omega=d \alpha / d t
$$

We will represent the equation of state for the Cauchy stress tensor in the form

$$
\mathbf{T}=-p \mathbf{E}+\mathbf{S}
$$

Then, taking relations (4.1) and (4.2) into account, the equations of motion and the incompressibility condition reduce to the form

$$
\begin{aligned}
& -\frac{\partial p}{\partial X}+\frac{\partial S_{11}}{\partial X}+\frac{\partial S_{21}}{\partial Y}=\rho \frac{d v_{1}}{d t}, \quad-\frac{\partial p}{\partial Y}+\frac{\partial S_{12}}{\partial X}+\frac{\partial S_{22}}{\partial Y}=\rho \frac{d v_{2}}{d t} \\
& \frac{\partial M_{13}}{\partial X}+\frac{\partial M_{23}}{\partial Y}+S_{12}-S_{21}=\gamma \frac{d^{2} \alpha}{d t^{2}}, \quad \frac{\partial v_{1}}{\partial X}+\frac{\partial v_{2}}{\partial Y}=0
\end{aligned}
$$


We will assume that in shear flow the velocity and angle of rotation have the form

$$
v_{1}=v(Y), v_{2}=0, \alpha=\alpha(Y)
$$

It can be shown that all the deformation rate tensors and flexural deformation tensors, apart from $\varepsilon$ in steady shear flow of the form (4.4), vanish. Taking expansion (1.23) into account this means that in flow (4.1) and (4.4) the dependence of the equations of state on the deformation prehistory reduces to the dependence on $B$ and $\epsilon$. In other words, in shear flow no viscoelastic liquid is distinguishable from a differential-type liquid of $(1,0)$ complexity.

The determination of the fields $v$ and $\alpha$ from Eqs (4.3) requires a more detailed specification of the equations of state. We will use the following dependences for $\mathbf{S}$ and $\mathbf{M}$

$$
\begin{aligned}
& \mathbf{S}=\mu_{1} \epsilon+\mu_{2} \epsilon^{T}-\left(v_{1} \mathbf{B}+v_{2} \mathbf{B}^{T}\right) \cdot \mathbf{B}^{T} \\
& \mathbf{M}=\eta_{1} x+\eta_{2} x^{T}+v_{1} \mathbf{B}+v_{2} \mathbf{B}^{T}
\end{aligned}
$$

where $\mu_{1}, \mu_{2}, v_{1}, v_{2}, \eta_{1}, \eta_{2}$ are constants.

Equations (4.5) represent a linear dependence of the stress and couple-stress tensors on the velocity tensors. In the equilibrium state the law of state (4.5) reduces to relations of the form (2.14). gives

Assuming that there are surface couple stresses $(\mathbf{N} \cdot \mathbf{M}=0$ when $Y=h$ ) the solution of system (4.3)

$$
\begin{aligned}
& T_{21}=\tau, \quad T_{22}=0, \quad M_{23}=\tau \xi(h-Y) \\
& \nu(Y)=v^{\rho} Y, \nu^{0}=\frac{\tau}{\mu_{1}} \\
& \alpha(Y)=\alpha^{0}+\frac{\tau}{v_{1}} \xi\left(h Y-\frac{Y^{2}}{2}\right) ; \quad \xi=\frac{\mu_{2}}{\mu_{1}}-1
\end{aligned}
$$

( $\alpha^{0}$ is the angle of orientation when $Y=0$ ).

Formulae (4.6) describe the linear distribution of velocities and couple stresses over the depth.

Flow in a channel. Consider the plane flow of a micropolar liquid in a layer between two parallel plates $(-\infty<X<\infty,-h<Y<h,-\infty<Z<\infty)$, due to a pressure drop in the direction of the $X$ axis.

We will take the velocity fields, the angle of rotation of the trihedron $\mathbf{D}_{k}$ and the pressure $p$ in the form

$$
v_{1}=v(Y), v_{2}=0, \alpha=\alpha(Y), p=-G X(G=\text { const })
$$

As in shear flow, in the flow represented by (4.7) the micropolar liquid is indistinguishable from a differential-type liquid of $(1,0)$ complexity.

For the law of state (4.5) the solution of Eqs (4.3) is given by the formulae

$$
\begin{aligned}
& T_{21}=-G Y, \quad T_{22}=-G X \\
& M_{23}=\frac{\xi G}{2} Y^{2}+\frac{\xi G}{6} h^{2}-\frac{v_{1}}{2 h}\left(\alpha_{+}-\alpha_{-}\right) \\
& v(Y)=\frac{G}{2 \mu_{1}}\left(h^{2}-Y^{2}\right) \\
& \alpha(Y)=-\frac{\xi G}{6 v_{1}} Y\left(Y^{2}-h^{2}\right)+\frac{1}{2 h}\left(\alpha_{+}-\alpha_{-}\right) Y+\frac{1}{2}\left(\alpha_{+}+\alpha_{-}\right)
\end{aligned}
$$

In (4.8) $\alpha_{ \pm}$are the angles of orientation of the trihedron on the plates $\left(\alpha( \pm h)=\alpha_{ \pm}\right)$.

Couette flow. Consider the flow of a viscoelastic micropolar liquid between rotating coaxial cylinders. Suppose the flow is given by the formulae

$$
\begin{aligned}
& \mathbf{v}=u(R) \mathbf{e}_{\Phi}, \omega=\frac{v(R)}{R} \mathbf{e}_{\mathrm{Z}}, p=p(R), \alpha=\alpha(R) \\
& \mathbf{D}_{1}=\mathbf{e}_{R} \cos \alpha+\mathbf{e}_{\Phi} \sin \alpha, D_{2}=-\mathbf{e}_{R} \sin \alpha+\mathbf{e}_{\Phi} \cos \alpha, D_{3}=\mathbf{e}_{Z}
\end{aligned}
$$


It can be shown that in this case the following equations are satisfied

$$
\begin{aligned}
& \mathbf{B}=\alpha^{\prime} \mathbf{e}_{R} \otimes \mathbf{e}_{Z}+\frac{1}{R} \mathbf{e}_{\Phi} \otimes \mathbf{e}_{Z}, \quad \epsilon=\left(\nu^{\prime}-\frac{v}{R}\right) \mathbf{e}_{R} \otimes \mathbf{e}_{\Phi}, \quad x=\left(\frac{v^{\prime}}{R}-\frac{v}{R^{2}}\right) \mathbf{e}_{R} \otimes \mathbf{e}_{Z} \\
& \mathbf{A}_{k}=\mathbf{B}_{k}=0(k=2,3, \ldots)
\end{aligned}
$$

The last two equations denote that, in the class of flows (4.9), an arbitrary viscoelastic micropolar liquid is indistinguishable from a differential-type liquid of complexity (1.1).

The equations of motion reduce to three equations in $v, \alpha$ and $p$

$$
\begin{aligned}
& -p^{\prime}+S_{R R}^{\prime}+\frac{S_{R R}-S_{\Phi \Phi}}{R}+\frac{\rho v^{2}}{R}=0, S_{R \Phi}^{\prime}+\frac{S_{R \Phi}+S_{\Phi R}}{R}=0 \\
& M_{R Z}^{\prime}+\frac{M_{R Z}}{R}+S_{R \Phi}-S_{\Phi R}=0
\end{aligned}
$$

In view of the complexity of the final expressions we will only present the solutions of (4.10) for the velocity field and the angle of rotation (we use constitutive relations (4.5))

$$
\begin{aligned}
& v(R)=c_{0} R+\frac{c_{1}}{2 \eta_{1}} R \ln \frac{R^{2}}{\delta+R^{2}}, \quad \alpha(R)=\alpha_{0}+c_{1} I(R)+c_{2} \ln \frac{R}{R_{0}} \\
& c_{0}=\Omega_{1}-\frac{c_{1}}{2 \eta_{1}} \ln \frac{R_{1}^{2}}{\delta+R_{1}^{2}} \\
& c_{1}=\frac{2 \eta_{1}\left(\Omega_{0}-\Omega_{1}\right)}{\ln \left[R_{0}^{2}\left(\delta+R_{1}^{2}\right)\right]-\ln \left[R_{1}^{2}\left(\delta+R_{0}^{2}\right)\right]} \\
& c_{2}=\left(\ln \frac{R_{1}}{R_{0}}\right)^{-1}\left[\alpha_{1}-\alpha_{0}-c_{1} I\left(R_{1}\right)\right] \\
& I(R)=\int_{R_{0}}^{R}\left[\frac{\xi}{2} \ln \left(\delta+R^{2}\right)-\frac{\delta}{\delta+R^{2}}\right] d R, \quad \delta=\frac{\eta_{1}}{\mu_{1}}
\end{aligned}
$$

In (4.11) $\Omega_{0}$ and $\Omega_{1}$ are the angular velocities of rotation of the cylinders between which the liquid moves, $R_{0}$ and $R_{1}$ are their radii $\left(R_{1}>R_{0}\right)$, and $\alpha_{0}$ and $\alpha_{1}$ are the angles of orientation of the trihedron of the director vectors on the cylinder surfaces.

Poiseuille flow. As an example of three-dimensional flow, consider the steady axisymmetrical flow of a viscoelastic micropolar liquid in a circular tube, due to a pressure drop. We will introduce the cylindrical coordinates $R, \Phi, Z$ so that the $Z$ axis coincides with the axis of symmetry of the tube. We will seek a solution in the form

$$
\begin{aligned}
& \mathbf{v}=v(R) \mathbf{e}_{Z}, \omega=0, p=-G Z+q(R), \alpha=\alpha(R) \\
& \mathbf{D}_{1}=\mathbf{e}_{R} \cos \alpha+\mathbf{e}_{Z} \sin \alpha, \mathbf{D}_{2}=\mathbf{e}_{\Phi}, \mathbf{D}_{3}=-\mathbf{e}_{R} \sin \alpha+\mathbf{e}_{Z} \cos \alpha
\end{aligned}
$$

In the flow represented by (4.12) all the deformation rate tensors and the flexural deformation tensor, apart from $\epsilon$, vanish. Taking (1.23) into account this means that, in a flow of the form (4.12), the dependences of the equations of state on the deformation prehistory consist of the dependences on $\mathbf{B}$ and $\epsilon$.

Using isotropicity of the functions $\mathbf{S}$ and $\mathbf{M}$, it can be shown that in flow of the type (4.12) the following components of the tensors $S$ and $M$ vanish: $S_{R \Phi}, S_{\Phi R}, S_{Z \Phi}, S_{\Phi Z}, M_{R R}, M_{\Phi \Phi}, M_{Z Z}, M_{R Z}, M_{Z R}$.

The equations of motion reduce to three equations in the unknowns $v, \alpha$ and $q$

$$
\begin{aligned}
& S_{R R}^{\prime}+\frac{S_{R R}-S_{\Phi \Phi}}{R}-q^{\prime}=0, \quad S_{R Z}^{\prime}+\frac{S_{R Z}}{R}+G=0 \\
& M_{R \Phi}^{\prime}+\frac{M_{R \Phi}+M_{\Phi R}}{R}+S_{Z R}-S_{R Z}=0
\end{aligned}
$$


Integration of these equations for constitutive relations (4.5) and the boundary conditions $v\left(R_{0}\right)=0$ and $\alpha\left(R_{0}\right)=\alpha_{0}$ when $R=R_{0}$ gives

$$
\nu(R)=\frac{G}{4 \mu_{1}}\left(R_{0}^{2}-R^{2}\right), \quad \alpha(R)=\alpha_{0}+\frac{\xi G}{18 v_{1}}\left(R_{0}^{3}-R^{3}\right)
$$

One can similarly investigate other flows, for example, annular flow between coaxial cylinders. This research was supported by the Russian Foundation for Basic Research (99-01-01019).

\section{REFERENCES}

1. AERO, E. L. and KUVSHINSKII, Ye. V., The fundamental equations of the theory of elasticity of media with rotational interaction of particles. Fiz. Tverd. Tela, 1960, 2, 7, 1399-1409.

2. KOITER, W. T., Couple-stresses in the theory of elasticity, Pt I-III. Procn. Koninkl. Neterland. Akad. Wetensh, 1964, 67, 1, $17-44$.

3. PAL'MOV, V. A., The fundamental equations of the theory of asymmetrical elasticity. Prikl. Mat. Mekh., 1964, 28, 3, 401-498.

4. TOUPIN, R. A., Theories of elasticity with couple-stress. Arch. Ration. Mech. and Analysis, 1964, 17, $2,85-112$.

5. SHKUTIN, L. I., Flexible Body Deformation Mechanics. Nauka, Novisibirsk, 1988.

6. ZHILIN, P. A., The fundamental equations of the non-classical theory of elastic shells. Trudy Leningr. Politekh. Inst., 1982, $386,29-46$.

7. ZUBOV, L. M., Variational principles and invariant integrals for non-linearly elastic bodies with couple stresses. Izv. $A$ kad. Nauk SSSR. MTT, 1990, 6, 10-16.

8. YERMEYEV, V. A. and ZUBOV, L. M., The stability of elastic bodies with couple stresses. Izv. Ross. Akad. Nauk. MTT, 1994, 3, 181-190.

9. ZUBOV, L. M., Nonlinear Theory of Dislocations and Disclinations in Elastic Bodies. Springer, Berlin, $1997,205$.

10. ERINGEN, A. C., Linear theory of micropolar viscoelasticity. Int. J. Eng. Sci. 1967, 5, 2, 191-204.

11. DeSILVA, C. N. and KLINE, K. A., Nonlinear constitutive equations for directed viscoelastic materials with memory. Z. Angew Math. and Phys., 1968, 19, 1, 128-139.

12. DINARIYEV, O. Yu. and NIKOLAYEVSKII, V. N., The constitutive relations for a viscoelastic medium with microrotations. Prikl. Mat. Mekh., 1997, 61, 6, 1023-1030.

13. AERO, E. L., BULYGIN, A. N. and KUVSHINSKII, Ye. V., Asymmetric hydromechanics. Prikl. Mat. Mekh., 1965, 29, 2 , 297-308.

14. ERINGEN, A. C., Theory of micropolar fluids. J. Math. Mech., 1966, 16, 1, 1-18.

15. MIGUN, N. P. and PROKHORENKO, P. P., Hydrodynamics and Heat Transfer of Gradient Flows of a Microstructural Liquid. Nauka i Tekhnika, Minsk, 1984.

16. BESSONOV, N. M. and AERO, E. L., The couple-stress hydrodynamic theory of friction. Treniye i Iznos, 1993, 14, 1, $107-111$.

17. TRUESDELL, C., A First Course in Rational Continuum Mechanics. Johns Hopkins University, Baltimore, MD, 1972.

18. ASTARITA, G. and MARRUCCI, G., Principles of Non-Newtonian Fluid Mechanics. McGraw-Hill, London, 1974.

19. LANDAU, L. D. and LIFSHITS, E. M., Theoretical Physics, Vol. 7, Theory of Elasticity. Nauka, Moscow, 1987.

20. AERO, E. L. and BULYGIN, A. N., Hydrodynamics of liquid crystals. In Advances in Science and Technology. Series Hydrodynamics, Vol. 7. Vsesoyuz. Nauch. i Tekh., Moscow, 1973.

21. CHANDRASEKHAR, S., Liquid Cystals. Cambridge: Cambridge University Press, 1974.

22. ERICKSEN, J., Equilibrium theory of liquid crystals. In Ericksen J., Research in Continuum Mechanics. Collected Works. Mir, Moscow, 1977, 46-123.

23. GENNES, P. G. de. The Physics of Liquid Crystals. Clarendon Press, Oxford, 1974.

24. BLINOV, L. M. and PIKIN, S. A., Liquid crystals. In Physics Encyclopedia, Vol. 2. Sov. Entsiklopediya, Moscow, $1990,31-36$.

25. FINN, R., Equilibrium Capillary Surfaces. Springer, New York, 1986.

26. YEREMEYEV, V. A. and ZUBOV, L. M., The conditions of phase equilibrium in non-linear elastic media with a microstructure. Dokl. Ross. Akad. Nauk, 1992, 322, 6, 1052-1056. 